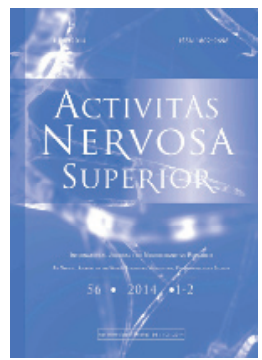

ANS: Journal for Neurocognitive Research Homepage:

WWW.activitas.org

IDEAS \& OPINION

\title{
THE ESSENCE OF FREE WILL AND OF ITS REAL MAIN RESTRICTION
}

\author{
Vadim Rotenberg* \\ Tel Aviv University, Bat-Yam, Israel
}

\begin{abstract}
Free will is actually a free choice of a definite type of behavior from many options that are determined by different objective conditions. This choice is the function of the Self-Image as the highest level of personality. At the same time Self-Image restricts the free will making it different from arbitrariness. The essence of the free will is the subject's responsible choice of one option of motivated behavior from many options and this choice is determined by the subject's Self-Image as the most integrative and not totally conscious or realized representation of the Self that includes the highest personal values.
\end{abstract}

Key words: Volitional choice; Arbitrariness; Personality; Self-Image

\section{INTRODUCTION}

In this short article I am going to discuss the role of the human's self estimation and personality in the realization of the free will. However, before turning to this topic it is necessary to elucidate the problem of the free will in general, whether it really takes place in our life and subject can behave according to his/her own intentions and wishes, or behavior is in any case totally determined by the objective conditions and different factors of the outward environment we are dealing with as well as by physiological mechanisms of the living organism. It is an old topic of discussion between philosophers and scientists.

\section{FREE WILL IN HUMANS AND ANIMALS - SIMILARITY AND DIFFERENCE}

On the one hand, when we are considering some simple volitional acts the presence of the free will in our actions looks out as an obvious. For instance, I wish to perform a definite gesture (to raise my hand) and I am doing it. I decide to go in the definite direction and I am going in this direction. However, if we are estimating such actions as a sign of our free will what is the essential difference between humans and animals? The behavior of animals is also often quite spontaneous and not always determined by conditional reflexes. If there are no obstacles, and no danger of being punished animal can run in any direction it is choosing in order to satisfy a pure interest in the environment, not only to search for food or to avoid threats. However, are we ready to agree that our free will we are so proud of is similar to the

*Correspondence to: Vadim Rotenberg, email: vadir@post.tau.ac.il

Received June 25, 2014; accepted July 10, 2014; Act Nerv Super 56(1-2), 52-55; ISSN-1802-9698 
"free will" of animals? Does such similarity not de-evaluating our own free will? Is in such context the free will of humans not loosing something essential?

On the other hand, although our behavior may be really free according to the goal we are choosing, according to the direction of our activity, in some aspects it is really like the behavior of animals being actually restricted by the peculiarities of numerous physiological mechanisms we need to use in order to perform our chosen actions. Many physiologists became rigidly fixed on these natural restrictions. Yes, in any case we are unable to perform actions beyond the opportunities and limitations provided, for instance, by our motor system or by brain functions. However, from my point of view to speak seriously about such limitations of our free will is no more relevant than to emphasize that even if we have a very strong intention we are unable to go though the brickwork, and to make according to this limitation a conclusion that the free will is absent. The absence of free behavior in the prison is also not the same as the absence of the free will because even in a prison subject can choose different types of real or at least virtual behavior.

\section{THE MODERN SCIENTIFIC APPROACH TO THE PROBLEM}

Thus, what is the real sign of human's free will? J. Kuhl and M. Quirin (2011) presented an excellent and comprehensive analysis of the nature of free will. According to these authors, volition is a context-sensitive form of top-down control of emotion, cognition and behavior. It is not a freedom from causal determination, but it is a free choice of the definite type of behavior from numerous available options each of them being causally determined by different objective conditions.

People are making this choice in front of many features of reality that are sometimes in contradictive interrelations and it can be difficult to find a definite algorhythm of their interrelationships. The more broad is the opportunity to accept options of the subject's relationships with the world and these determining factors the higher is the level of freedom (of the choice from these options).

To such determining factors belong our attitudes to the world formed in the process of maturation and of the development of our personality, our relationships with the surrounding people and with virtual images determined by our spiritual life, our present actual needs etc. To be free does not mean to ignore all these factors. Such ignoring reflects not a freedom but arbitrariness. Free will represents itself in the ability to select the definite goal and to integrate the holistic behavior oriented on this goal while taking into consideration many factors. In order to realize a free will it is necessary to search for the most relevant options from the big set of different options

J.Kuhl and M. Quirin emphasized that some biologists belief that the absence of the free will is confirmed by the well known physiological investigations that show that the activity of the definite brain zones that reflect the predisposition of the brain to perform movement starts even before the subject comes to the conscious decision to perform this movement. From the point of view of these biologists it means that the decision to perform movement is not a decision of the Self, but the decision of the brain, and consequently it is a pure physiological process not related to the free will.

\section{THE KEY ROLE OF THE SELF-IMAGE}

However, this conclusion does not take into consideration that the highest level of our personality is our Self-Image (Rotenberg, 2012) as a function of the frontal lobe of the right hemisphere. In contrast to the Self-Concept that is related to the activity of the left frontal lobe and is totally available for the conscious realization being relatively simple and monosemantic, Self-Image is polysemantic, it includes and integrates many views and 
intentions that are contradictive from the logical point of view and from this reason cannot be fully realized.

Being the highest level of our Self, Self-Image is not totally consciously realized but it is not a part of our subconscious - it is, let us say, a superconscious that includes also all conscious attitudes and integrates them with attitudes that may be in contradictions to our conscious Self-Concept. Our free will in order not to be in confrontation with our self must correspond to our Self-Image even more that to our Self-Concept, and in this condition the preparation to the movement that corresponds to the attitudes and intentions of our SelfImage can start before our conscious realization of these intentions in the stressful and dangerous conditions (for instance during car driving ) when many relevant acts have to be performed without their conscious realization because they must be performed immediately and subject has no time for their analysis and conscious decision. However it is not a pure automatic activity like a reflex. These acts are performed by the participation of Self-Image and include the free will to avoid the threat. But they can look out from the outside (and also from the point of Self-Concept) as if being performed automatically by relatively simple brain mechanisms.

The formation of the Self-Image is the highest function of the human's right hemisphere, of its frontal lobe. Right hemisphere in general is characterized by the openness to all numerous experiences in the past and present including virtual experiences evolved by the culture, but they do not cause a chaotic mixture because at the same time the right hemisphere frontal lobe is able to integrate all these experiences in the holistic entity. It is exactly the function of the Self-Image. The more open is the subject to the relevant information, the more broad are the options for the choice of a relevant behavior, however only if all different elements of information became integrated.

It is necessary to take into consideration that the Self-Image includes the highest personal values that prevent the destructive behavior.

According to J. Kuhl and M. Quirin self-determination does not disrupt the conception of causal world although it relates to a complex level of causation (this complexity of the causal factors opens the opportunity for choice what is the main sign of the free will). Choosing among behavioral options after considering relevant input information is performed on the highest level of personality functioning. Freedom is a relieving of constraints placed by external forces but freedom of choice must be relevant to the subject's self.

J. Kuhl and M.Quirin emphasizes that excessive stress reduces accessibility of high-level systems (in my term, of Self-Image). I suppose that it happens if the stress causes neurotic anxiety, learned helplessness and depression all these disorders being characterized by renunciation of search (Rotenberg, 2009). Such stress restricts behavior and overcomes the ability of the highest right hemisphere functions to integrate the contradictive information and contradictive behavioral attitudes into a holistic system.

It is impossible to control all factors at once however the choice of the definite goal determines the direction of search of those factors that have to be controlled. This free choice includes also the readiness to be responsible for it and this readiness also distinguishes the choice according to the free will from the arbitrariness.

It is also necessary to take into consideration that the difference between the free will and arbitrariness lies not only in the deficiency of the probability forecast of the outcome of the arbitrary selected behavior. A mistaken prognosis may be made even when the behavior is based on free will, but arbitrariness in contrast to the free will reflects the absence of attitudes towards such prognosis, absence of attempts to perform it, renunciation of search for factors that may influence the outcome of activity. Objective factors that partly determine the outcome of behavior do not disappear when being ignored, but such ignorance makes behavior chaotic and destructive.

Thus, the free will is the free choice of a definite line of behavior from many different available lines. Every such line has its own determination. The free will is a choice of one out 
of numerous options but every such option has its own determination by many factors that are in interrelationships with each other.

Finally, it is very important to take into consideration that this volitional choice is not unrestricted. Maybe it looks paradoxically at first glance, but it is restricted by the subject's holistic view of himself, of his personality, it means by the same Self-Image. Exactly such restriction of the free will by Self-Image makes free will different from arbitrariness and at the same time differ humans from animals who have only a physical feeling of himself but have no Self-Image as an integrative view of himself.

A person can decide to be free from many conditions but he/she cannot be free from him/her-self when the Self-Image is formed. He/she has to be in peace with him/her-self, with self representation that really determines restrictions of our choice between different opportunities of behavior.

Here is a clear example of such restriction. A very creative and well-known Russian poet Bulat Okudjava was invited by a high level Soviet official who applied to him with a proposition that was inappropriate for the poet. To disagree with such officials was dangerous, however the answer of Okudjava was: “No, I do not agree. I see you for the first and probably also for the last time, while with myself, with my personality I have to spend all my life". This answer is a demonstration of the inner free will restricted only by the SelfImage.

If for the subject all different and conflicting decisions are equal, it means that they all are senseless. A real sense has only the choice that is acceptable for the personality, for the SelfImage. If a person does not have such feeling of himself, if Self-Image is not holistic and definite he/she really has nothing to do with the "free will." Then a person may feel that such unrestricted free will may cause destructive behavior and being afraid of it, he/she may choose a dependence on somebody else.

\section{CONCLUSION}

Thus, the essence of the free will is the subject's choice of one option of motivated behavior from many options and this choice is determined by the subject's Self-Image as the most integrative and not totally realized representation of the Self.

\section{REFERENCES}

Kuhl, J. \& Quirin, M. (2011). Seven steps toward freedom and two ways to lose it. Overcoming limitations of intentionality through Self-confrontational coping with stress. Social Psychology, 42, 7484.

Rotenberg, V.S. (2009). Search activity concept: Relationship between behavior, health and brain functions. Activitas Nervosa Superior, 51, 12-44.

Rotenberg, V. S. (2012). Two high levels of self-identification in relationships to consciousness, social motives and brain laterality. Activitas Nervosa Superior, 54, 77-83. 